\title{
Characteristics of Invasive Pneumococcal Disease in Young Children before the Introduction of PCV13 in Lombardy, Italy $^{*}$
}

\author{
Giovanni Radaelli $^{1,2 \#}$, Enrica Riva ${ }^{1}$, Marcello Giovannini ${ }^{1}$ \\ ${ }^{1}$ Department of Pediatrics, San Paolo Hospital, University of Milan, Milan, Italy; ${ }^{2}$ Unit of Medical Statistics, San Paolo Hospital, \\ University of Milan, Milan, Italy. \\ Email: " giovanni.radaelli@unimi.it
}

Received May $4^{\text {th }}, 2012$; revised June $5^{\text {th }}, 2012$; accepted July $8^{\text {th }}, 2012$

\begin{abstract}
An active surveillance system of invasive pneumococcal disease (IPD) started on September 2008 in Lombardy, Italy, among children aged less than 60 months and admitted for suspicion of IPD at emergency room of ten hospitals. This study examined the clinical characteristics of children enrolled up to December 2010, that is just before the introduction in this region of voluntary mass vaccination, free of charge, based on the 13-valent pneumococcal conjugate vaccine (PCV13). Two hundred fifty one children were suspected and 20 were confirmed as having IPD, based on isolation of Streptococcus pneumoniae from blood. Thirty-nine percent of children had received pneumococcal vaccination previously, and full vaccination with three doses of hepta-valent vaccine (PCV7) had been administered in 21.4\%. Co-morbidity conditions were more frequent in children with confirmed than non-confirmed IPD (10.0\% vs. $0.9 \%)$. The annual incidence rate of confirmed IPD was 28.6/100,000 (binomial 95\% confidence interval, 18.6 to 44.1/100,000). Among confirmed IPD children, 11 exhibited pneumonia with bacteremia, 6 bacteremia without focus, 2 septicemia, 1 meningitis. Seventeen (85\%) isolates were identified, and nine serotypes. The overall serotype coverage was $29.4 \%$ for PCV7 and $82.3 \%$ for PCV13. In non-vaccinated children, the coverage of PCV7 and PCV13 was $41.7 \%$ and $75.0 \%$, respecttively. Non-vaccine serotypes 12B, 15C, and 23B were identified. Antibiotic resistance was found in seven children, that is against penicillin (serotype 15C), erythromycin (14,19A, 19F), tetracycline (15C, 19F), chloramphenicol (23F), and trimethoprim-sulfamethoxazole $(23 \mathrm{~F})$. Two of these children had received antibiotic therapy (penicillin or azithromycin) during the week before hospital admission. The coverage vaccination rate in Lombardy was relatively low during the surveillance period and serotype distribution widespread. The introduction of PCV13 and a mass vaccination program in young children might impact positively on invasive pneumococcal disease in this surveilled population. Active long-term surveillance of non-vaccine serotypes is required wordwide.
\end{abstract}

Keywords: Streptococcus pneumoniae; Invasive Pneumococcal Disease; Serotype; Pneumococcal Vaccine; Children

\section{Introduction}

Streptococcus pneumoniae (S. pneumoniae) is a leading cause of invasive infection and vaccine-preventable deaths among young children [1-4]. Decline in invasive pneumococcal disease (IPD) has been observed in countries which introduced the heptavalent pneumococcal conjugate vaccine (PCV7) in their immunization programs [5-6], with higher reduction in the US, where also decline in IPD mortality occurred [7]. While pneumococcal serotypes may vary across geographical locations, also resulting in different vaccine coverage $[2,8]$, non-

\footnotetext{
*The Invasive Pneumococcal Disease Lombardy Study Group (see Appendix A).

${ }^{\#}$ Corresponding author.
}

vaccine serotype replacement may emerge worldwide $[9,10]$. The World Health Organization recommends to monitor actively the effect of pneumococcal vaccination [1].

In Italy, few local studies have been conducted in the past in children aged less than 60 months [11-15]. Recently Azzari et al. [16] reported merged national data on Italian children aged $0-16$ years, which however focused on serotype coverage only. The heptavalent pneumococcal conjugate vaccine PCV7 was licensed in Italy in 2001 and progressively introduced since 2003, recommended firstly in at-risk children aged less than 5 years and then planned on different regional vaccination strategies. This setting has contributed to make broad difference in the vaccination coverage across geographi- 
cal areas. In Lombardy, PCV7 was initially offered to at-risk children free of charge, otherwise available at the private market on voluntary demand with co-payment of about USD65 per dose up to December 2009. Based on the current national plan for vaccinations [17] and regional directives, the pneumococcal conjugate vaccine PCV13 is now offered in Lombardy on voluntary demand and free of charge, and vaccination has been scheduled in infants to be administered at three single doses at 3, 5 - 6, and $11-12$ months of age [17].

Indeed, while Lombardy is a crucial region with around $10,000,000$ resident people in 2011, the characteristics of IPD in young children, including the pneumococcal serotype distribution, have been never actively investigated.

The main objective of this study was to describe the clinical characteristics of the invasive pneumococcal disease in young children residing in the North-West Lombardy, Italy, and to determine the serotype distribution of S. pneumoniae occurring just before the introduction of the PCV13, that was licensed in Italy for commercialization on April 30, 2010.

These data would provide general epidemiological information useful to better examine the traits of invasive pneumococcal disease in young children, and further to help the Health Authorities and industry for hopefully improving immunization strategies.

\section{Methods}

\subsection{Setting}

This study is a part of an active surveillance of IPD in young children that started on September 01, 2008, in North-West Lombardy, Italy, also including the municipality of Milan. Cases of suspected IPD were collected from ten hospitals homogeneously located in the area delimited by four Reference Local Health Authorities districts. This study analyzed children enrolled up to December 31,2010, that is before of the introduction of a new regular vaccination strategy and implementation of the PCV13 in Lombardy. The scrutinized area comprised at the beginning of the surveillance around $3,500,000$ people, including 130,000 children aged less than 60 months, of whom 30,000 (12,000 aged less than 24 months) referenced to the participant hospitals.

\subsection{Subjects}

Children admitted at emergency room of the hospitals were assessed for eligibility. Inclusion criteria were: age at admission 28 days - 59 months; being residing in the monitored area; suspicion of IPD, that is any S. pneumoniae clinical syndrome; negative urinalysis for urinary tract infections based on fast urine test; $\mathrm{C}$-reactive pro- tein $>15 \mathrm{mg} / \mathrm{L}$. The additional inclusion criterion and/or body (rectal) temperature $\geq 38^{\circ} \mathrm{C}$ was considered in children aged $\leq 36$ months, as it may be per se suggestive of IPD within this range of age [18]. The exclusion criterion was refusal of parents or the legal guardian to give written consent. Urinalysis was performed by Aution sticks10EA (ARKRAY Europe, B.V., Amstelveen, The Netherlands). C-reactive protein was measured by direct immunoturbidimetry (Tina-Quant, Roche Diagnostics, Hoffmann-La Roche Ltd, Basel, Switzerland).

\subsection{Ethics}

The study was carried out in accordance with the Declaration of Helsinki and Good Clinical Practice guidelines. The ethics committee of each hospital approved the study protocol. Written informed consent was obtained from the parents or legal guardian after the study had been fully explained.

\subsection{Case Definition}

A case of confirmed IPD was defined in accordance with the Centers for Disease Control and Prevention (Atlanta, GA, USA) [19,20]. Definition of specific IPD syndrome was in accordance with the International Classification of Diseases-9 [21].

\subsection{Identification of Isolates}

Blood samples were taken in children within $24 \mathrm{~h}$ of recruitment. Cerebrospinal fluid samples were additionally taken in children with clinical syndrome of sepsis and/or meningitis for confirmation. S. pneumoniae isolates were identified by standardized laboratory procedures. Fresh cultures of isolates on blood agar plates and in Amies medium swab were sent to the central reference laboratory (Milan, Italy) for analysis and stored on beads at $-70^{\circ} \mathrm{C}$. Isolates were then confirmed for S. pneumoniae by testing for alpha haemolysis on blood agar, optochin susceptibility and bile solubility.

\subsection{Serotyping and Antimicrobial Susceptibility}

Serotyping was based on Omni serum (Statens Serum Institut, Copenhagen, Denmark) that includes antibodies to all recognized S. pneumoniae serotypes. A suspension of the test organism was prepared in saline solution $0.9 \%$ from well isolated colonies grown on sheep blood agar plates for 18 to $24 \mathrm{~h}$ in $5 \% \mathrm{CO}_{2}$ at $35^{\circ} \mathrm{C}$. One drop of this suspension was mixed with 1 drop of antiserum and 1 drop of methylene blue and, after incubation at room temperature for $10 \mathrm{~min}$, examined at $1000 \times$ magnification. Visible evidence of capsular swelling with Omni serum, i.e., a positive Quellung reaction, was considered 
positive, and followed by type specific antisera. Antimicrobial susceptibility against penicillin G, ceftriaxone, erythromycin, tetracycline, trimethoprim-sulfamethoxazole, chloramphenicol, levofloxacin, linezolid and vancomycin was performed by the E test method (AB Biodisk, Solana, Sweden) on Mueller-Hinton agar supplemented with 5\% defibrinated sheep blood (bioMérieux Italia Spa, Bagno a Ripoli, Italy). The minimal inhibitory concentration was defined in accordance with the Clinical and Laboratory Standards Institute breakpoints [22]. S. pneumoniae ATCC 49619 was used as control strain [22].

\subsection{Statistical Analysis}

Comparison between independent groups was performed by the Chi-square or Fisher's exact test or Mann-Whitney $\mathrm{U}$ test, as appropriate. Significance was posed at $P<$ 0.05 (two-tails). The statistical analyses were done using the SPSS software, version 17.0 for Windows (SPSS Inc.,
Chicago, IL).

\section{Results}

Two hundred fifty one children (140 boys, 111 girls; mean age [SD] 27.5 [16.8] months) were recruited. Blood testing data were available in 236 cases, and 20 (13 boys, 7 girls) were confirmed as having IPD. All of them were hospitalized. No fatality outcome occurred during hospitalization. The pooled annual incidence rate of confirmed IPD was 28.6/100,000 (binomial 95\% confidence interval, 18.6 to $44.1 / 100,000)$, that is $30.0 / 100,000(16.0$ to $46.9 / 100,000)$ in infants and $26.2 / 100,000(14.8$ to $48.9 / 100,000)$ in children aged $2-4$ years.

Table 1 reports the baseline characteristics of children by IPD status. The rate of co-morbidity conditions was higher in children with confirmed than non-confirmed (suspected) IPD. No child was human immunodeficiency virus positive.

Table 1. Baseline characteristics of the examined children, by IPD status.

\begin{tabular}{|c|c|c|c|c|}
\hline \multirow{2}{*}{ Characteristic } & \multirow{2}{*}{ All recruited $(\mathrm{n}=251)$} & \multicolumn{2}{|c|}{ IPD } & \multirow{2}{*}{$P$-value ${ }^{\dagger}$} \\
\hline & & Non-confirmed $(\mathrm{n}=216)$ & Confirmed $(\mathrm{n}=20)$ & \\
\hline \multicolumn{5}{|l|}{ Age (y) } \\
\hline$<2$ & $119(47.4)$ & $104(48.1)$ & $9(45.0)$ & \multirow[t]{2}{*}{0.787} \\
\hline $2-4$ & $142(52.6)$ & $112(51.9)$ & $11(55.0)$ & \\
\hline Gender (male) & $140(55.8)$ & $116(53.7)$ & $13(65.0)$ & 0.332 \\
\hline Race $(\text { white })^{\dagger \dagger}$ & $204(81.3)$ & $176(81.5)$ & $18(90.0)$ & 0.541 \\
\hline Having been breastfed (yes) & $198(78.9)$ & $175(81.0)$ & $15(75.0)$ & 0.555 \\
\hline Siblings (yes) & $125(49.8)$ & $110(50.9)$ & $8(40.0)$ & 0.350 \\
\hline Having a parent smoking (yes) & $76(23.0)$ & $70(32.2)$ & $5(25.0)$ & 0.620 \\
\hline Attending a community (yes) & $106(42.4)$ & $90(41.9)$ & $12(60.0)$ & 0.117 \\
\hline Congenital disease & $11(4.4)$ & $10(4.6)$ & $1(5.0)$ & 1.00 \\
\hline Co-morbidity conditions ${ }^{\dagger \dagger \dagger}$ (yes) & $4(1.6)$ & $2(0.9)$ & $2(10.0)$ & $0.037^{*}$ \\
\hline Having received pneumococcal vaccine before (yes) & $98(39.0)$ & $86(39.8)$ & $6(30.0)$ & 0.389 \\
\hline Type (conjugate/polysaccharide) & $87 / 11$ & $76 / 10$ & $5 / 1$ & 0.545 \\
\hline \multicolumn{5}{|l|}{ Doses $(\mathrm{n})^{\dagger+\dagger \dagger}$} \\
\hline 1 & $25(25.5)$ & $24(27.9)$ & $1(16.7)$ & \multirow{4}{*}{0.242} \\
\hline 2 & $48(49.0)$ & $43(50.0)$ & $2(33.3)$ & \\
\hline 3 & $21(21.4)$ & $15(17.4)$ & $3(50.0)$ & \\
\hline 4 & $4(4.1)$ & $4(4.7)$ & $0(0.0)$ & \\
\hline Median (range) age at the first dose of vaccine $(\mathrm{mo})^{\dagger+i \dagger}$ & $5.5(2-40)$ & $5.0(2-36)$ & $4.5(3-40)$ & 0.540 \\
\hline $\begin{array}{l}\text { Having received antibiotic therapy during the week } \\
\text { before recruitment (yes) }\end{array}$ & $86(34.3)$ & $79(36.6)$ & $2(10.0)$ & $0.015^{*}$ \\
\hline
\end{tabular}

Data are number of children (percentage within group) of children, except age at the first vaccination. 'Significance of comparison between IPD groups. (Chi-square test, exact Fisher's test or Mann-Whitney U test). "Statistically significant. ${ }^{\dagger}$ Non-white races include: Afro/Afro-American $(\mathrm{n}=24)$, Asian $(\mathrm{n}=7)$, other or mixed-race $(\mathrm{n}=16){ }^{\dagger \dagger}$ Immunodeficiency $(\mathrm{n}=1)$, sickle cell anemia $(\mathrm{n}=2)$, cardiovascular disease and/or chronic liver disease $(\mathrm{n}=1)$. ${ }^{\dagger \dagger \dagger}$ In children who received pneumococcal vaccine. 
Table 2 reports the clinical characteristics of children by IPD status. No significant difference occurred between children with confirmed or non-confirmed (suspected) IPD, except for clinical syndrome $(P<0.0001)$. In the IPD group, thoracic radiography was negative in 6 $(30.0 \%)$ children, and revealed lobar or bronchial pneumonia in respectively $5(25.0 \%)$ and $9(45.0 \%)$ children; the distribution of clinical syndrome differed between infants and children aged $2-4$ years $(P=016)$, that is: pneumonia $22.2 \%$ vs. $81.8 \%$, bacteremia $44.4 \%$ vs. $18.2 \%$, septicemia $22.2 \%$ vs. $0 \%$, meningitis $11.1 \%$ vs. $0 \%$. Blood leukocytes were higher in infants than children aged 2 - 4 years (median [range] 27.7 [17.1 - 41.0] vs. $\left.20.2[2.1-26.4] \times 10^{9} / \mathrm{L} ; P=0.023\right)$.

Table 3 shows the serotype distribution of IPD isolates by vaccination status. The PCV13 serotypes $1,7 \mathrm{~F}$, 19 A were the most frequently observed $(52.9 \%)$ of identified serotypes. Five of them were found in children who had received respectively one (one case, age 55 months, serotype 1), two (one case, age 37 months, serotype 19A) or three (3 cases, age 24 months, serotype 1; age 27 months, serotype 7F; age 15 months, serotype 19A) doses of PCV7. PCV7 serotypes 14, 19F, 23F were found in 5/14 (35.7\%) of non-vaccinated children. Serotypes $12 \mathrm{~B}, 23 \mathrm{~B}$ and $15 \mathrm{C}$, not included in any pneumo- coccal vaccine, were identified in non-vaccinated infants. Seven $(41.1 \%)$ of typified isolates showed antibiotic resistance. Resistance was found against: penicillin (one case; serotype $15 \mathrm{C}$ ), erythromycin ( 5 cases; serotypes 14 , 19A, 19F $[\mathrm{n}=2] ; 23 \mathrm{~F}$ ), tetracycline (4 cases; serotypes $15 \mathrm{C}, 19 \mathrm{~F}[\mathrm{n}=2], 23 \mathrm{~F}$ ), chloramphenicol (one case; 23F), and trimethoprim-sulfamethoxazole (one case; serotype 23F). Multiple resistance was found for serotypes 15C, $19 \mathrm{~F}$, and 23F. Two of seven children who exhibited a serotype with any antibiotic resistance had received antibiotic therapy during the week before hospital admission, and were affected by pneumonia. One child ( 35 months) had received penicillin and showed serotype 19F resistant to erythromycin and tetracycline. One child (41 months) had received azithromycin and showed serotype $23 \mathrm{~F}$ resistant against erythromycin, chloramphenicol and trimethoprim-sulfamethoxazole.

Serotypes 1, 7F, 14, 19F, 23F caused pneumonia, 19A, 19F, 23B bacteremia without an obvious focus, 12B and $23 \mathrm{~B}$ septicemia, and serotype $15 \mathrm{C}$ caused meningitis.

The overall expected serotype coverage rate, estimated on typified IPD cases, was $29.4 \%$ for PCV7 and $82.3 \%$ for PCV13. In non-vaccinated children, the coverage of PCV7 and PCV13 was $41.7 \%$ and $75.0 \%$, respectively.

Table 2. Clinical characteristics at hospital admission of children and syndrome, by IPD status.

\begin{tabular}{|c|c|c|c|c|}
\hline \multirow{2}{*}{ Characteristic } & \multirow{2}{*}{ All recruited $(n=251)$} & \multicolumn{2}{|c|}{ IPD } & \multirow{2}{*}{$P$-value } \\
\hline & & Non-confirmed $(\mathrm{n}=216)$ & Confirmed $(\mathrm{n}=20)$ & \\
\hline \multicolumn{5}{|c|}{ Body (rectal) temperature $\left({ }^{\circ} \mathrm{C}\right)$} \\
\hline Median (range) & $38.9(36.3-41.0)$ & $38.5(36.3-41.0)$ & $39.0(38.5-39.7)$ & 0.438 \\
\hline$\geq 38^{\circ} \mathrm{C}$ (yes) & $212(84.5)$ & $182(84.3)$ & $18(90.3)$ & 0.747 \\
\hline \multicolumn{5}{|c|}{ Blood leukocytes $\left(\times 10^{9} / \mathrm{L}\right)$} \\
\hline Median (range) & $19.1(2.1-50.3)$ & $19.1(2.6-50.3)$ & $21.0(2.1-41.0)$ & 0.165 \\
\hline \multicolumn{5}{|c|}{ C-reactive protein $(\mathrm{mg} / \mathrm{L})$} \\
\hline Median (range) & $90.2(2.1-450.0)$ & $91.1(5.0-450.0)$ & $140.9(4.1-400.0)$ & 0.146 \\
\hline$\leq 10$ & $11(4.4)$ & $5(2.3)$ & $1(0.5)$ & \\
\hline $11-40$ & $59(23.7)$ & $51(23.6)$ & $3(15.0)$ & \\
\hline $41-200$ & $126(50.6)$ & $114(52.8)$ & $10(50.0)$ & \\
\hline$>200$ & $53(21.3)$ & $46(21.3)$ & $6(30.0)$ & \\
\hline Syndrome (ICD-9) & & & & $<0.0001$ \\
\hline Pneumonia & $167(67.1)$ & $146(67.6)$ & $11(55.0)$ & \\
\hline Bacteremia & $6(2.4)$ & $0(0.0)$ & $6(30.0)$ & \\
\hline Septicemia & $4(1.6)$ & $2(0.9)$ & $2(10.0)$ & \\
\hline Meningitis & $2(0.8)$ & $1(0.5)$ & $1(5.0)$ & \\
\hline Other & $70(28.1)$ & $67(31.0)$ & & \\
\hline
\end{tabular}

Data are number of children (percentage within group) or median (range). Percentages may not sum to 100 because of rounding. ${ }^{\dagger}$ Significance of comparison between IPD groups (Chi-square test, exact Fisher's test or Mann-Whitney U test). 
Table 3. Serotype distribution of $S$. pneumoniae typified isolates in children with confirmed IPD by status of pneumococcal vaccination.

\begin{tabular}{|c|c|c|c|}
\hline \multirow{2}{*}{ Serotype } & \multicolumn{3}{|c|}{ Having received pneumococcal vaccination before } \\
\hline & All confirmed IPD $(n=20)$ & Yes $(n=6)$ & No $(n=14)$ \\
\hline Included in PCV7/PCV13 & $5[2,3](5)$ & & $5[2,3](5)$ \\
\hline 14 & $1[1,0](1)$ & & $1[1,0](1)$ \\
\hline $19 \mathrm{~F}$ & $2[0,2](2)$ & & $2[0,2](2)$ \\
\hline $23 \mathrm{~F}$ & $2[1,1](2)$ & & $2[1,1](2)$ \\
\hline Included in PCV13 & $9[3,6](1)$ & $5[1,4]$ & $4[2,2](1)$ \\
\hline 1 & $2[0,2]$ & $2[0,2]$ & \\
\hline $7 \mathrm{~F}$ & $4[1,3]$ & $1[0,1]$ & $3[1,2]$ \\
\hline $19 \mathrm{~A}$ & $3[2,1](1)$ & $2[1,1]$ & $1[1,0]$ \\
\hline Not included in any pneumococcal vaccine & $3[2,1](1)$ & & $3[2,1](1)$ \\
\hline $12 \mathrm{~B}$ & $1[1,0]$ & & $1[1,0]$ \\
\hline $15 \mathrm{C}$ & $1[1,0](1)$ & & $1[1,0](1)$ \\
\hline $23 \mathrm{~B}$ & $1[1,0]$ & & $1[1,0]$ \\
\hline Not available $^{\mathrm{a}}$ & $3[1,2]$ & $1[1,0]$ & $2[0,2]$ \\
\hline
\end{tabular}

Data are number of cases. Number of children aged $<24$ or $24-59$ months within square brackets. Number of cases showing any antibiotic resistance within round brackets, when different from zero. ${ }^{\mathrm{a}}$ Not available $=2$ non-viable cultures, 1 blood sample damaged.

\section{Discussion}

In the monitored area, before the introduction of PCV13, around $25 \%$ of confirmed IPD were caused by PCV7 serotypes, a proportion that is comparable with recent data from some European countries (e.g. 17\% in Portugal [23]) but is higher than in the US (around 2 - 3\% [5,24]). As pointed out by Aguiar et al. [23], a major factor for the difference may be the different vaccination programs adopted across geographical locations. Indeed, in this study $39 \%$ of children with suspected IPD had received pneumococcal vaccination, and full vaccination with three doses of PCV7 was administered in $21.4 \%$ of children only. While this is a relatively low vaccination rate, it should be noted that a report from the National Department of Health revealed at the time of starting this study difference in PVC7 coverage among different Italian regions, ranging from 20\% (Campania, South) to $95.2 \%$ (Emilia-Romagna, North), with a value of $27 \%$ in Lombardy [25].

The estimated annual incidence of IPD in children aged less than 5 years was $28.6 / 100,000$, that is similar to what has been reported in previuos Italian studies [11-14], and also comparable with data from the Active Bacterial Core Surveillance, an active population- and laboratorybased surveillance system in US, that estimated in 2010 an incidence of about 25 cases per 100,000 in the population aged 0 - 4 years [26]. In Lombardy, the new strategy adopted on July 2010 about voluntary mass pneu- mococcal vaccination offered free of charge in all children, and availability of PCV13, may be expected to impact positively on the coverage pneumococcal vaccination and IPD in young children. This active surveillance will provide in few years additional valuable data to appraise accurately effectiveness of this recent vaccine.

Regarding the serotype distribution, serotypes $7 \mathrm{~F}$ and 19A caused the majority of IPD, as also observed in the US [5,24]). This result is comparable with national data that estimated $19.2 \%$ of pneumococcal meningitis or sepsis occurred among children aged less than 5 years during in 2009 in Italy being caused by the serotype 19A [27]. A study conducted in Massachusetts showed throughout 2001-2007 period an increase in frequency of serotype 19A, that was the most cause of identified IPD cases in children (28\%) [28]. The relevance of this serotype, included in PCV13 but not in PCV7, has been as well indicated in South-West Asian countries [e.g., 29], and emphasized recently $[30,31]$.

Based on the results, the expected overall serotype coverage of PCV13 (82.3\%) would be within the range of values reported in the literature $([10,23])$. Recently, Azzari et al. [16] estimated in Italy a national pooled serotype coverage of $19.4 \%$ and $94 \%$ for PCV7 and PCV13, respectively. However, it should be noted that in the present study, heterogeneity in the serotype distribution of IPD was found, with a proportion of around 15\% of serotypes not included in any pneumococcal vaccine, as also observed recently in Aguiar et al. [23]. 
Concerning antimicrobial susceptibility, while the PCV7 serotypes accounted for the majority of erythromycin resistant isolates, consistently with recent literature [23], the only penicillin resistant serotype was $15 \mathrm{C}$. Moreover, it may be interestingly noted that two of the seven children who had received antibiotic therapy during the week before hospital admission exhibited serotype 19F and $23 \mathrm{~F}$ both resistant against erythromycin.

Lastly, it may be of interest to point out that, in this study, baseline clinical conditions at hospital admission did not significantly differ between children with confirmed and non confirmed (suspected) IPD, except for presence of higher co-morbidity in confirmed IPD cases. Moreover, rectal temperature ad hospital admission was $38^{\circ} \mathrm{C}$ or higher in about $85 \%$ of children. Although this result may be not unexpected it supports about value of the US approach to perform blood culture for every febrile young child.

This study has the major limitation of the small sample size of IPD cases, when compared with a recent report examining 144 pediatric hospitalized cases with IPD and identified serotype from 83 centers in 19 of 20 Italian regions [16]. Crudely, it may seem therefore of limited novelty, reporting data that may be not unexpected. However, it should be noted that in their study, Azzari et al. [16] included from Lombardy even fewer cases. Another limitation is that real-time-PCR analysis was not performed. Indeed, while use of molecular-based methods for the diagnosis of bacterial infections in blood is appealing and useful in research, real-time-PCR with blood samples for diagnosis of IPD may lack the sensitivity and specificity necessary for clinical practice [32]. Anyway, this study may have some strengths that might make it interesting and unique. Firstly, it is the only active surveillance of IPD implemented in Lombardy, that additionally evaluated the status of IPD up to the introduction of PCV13 in a European geographic region of high population density, for which few data exist. These data may therefore report useful information to the international scientific community, health authorities and industry for improving immunization pneumococcal strategies. Secondly, it would give a basis for a subsequent meaningful analysis about the impact of PCV13 and potential serotype replacement process.

\section{Conclusion}

As a whole, within the limitations of this study, one can conclude that PCV13 mass vaccination in Lombardy young children could positively impact on IPD. The dynamical role of non-vaccine $S$. pneumoniae serotypes, that might potentially limit the vaccines effectiveness, should be monitored through long-term active surveillance, hopefully coordinated across international coun- tries.

\section{Funding}

This is a sponsored study funded by Pfizer Italia (Protocol number 0887X1-4431).

\section{Conflicts of Interest}

GR is shareholder of Pfizer Inc.. All other Authors declare they have no conflict of interest.

\section{Authors' Contributions}

All authors made substantial contribution to the conception and design of the study, and in drafting the article. Additionally, GR performed the statistical analysis and supervised critically the final version of the article. All Authors approved the final manuscript.

\section{Acknowledgements}

We are sincerely grateful to Maria Laura Garlaschi and Laura Daprai of Pneumococcal Reference Laboratory in Lombardy, Milan, Italy. The Authors also thank the participating children and their families, and hospitals' staff involved in this research.

\section{REFERENCES}

[1] World Health Organization (WHO), "Position Paper on Pneumococcal Vaccines," The Weekly Epidemiological Record, Vol. 14, No. 87, 2012, pp. 129-144. http://www.who.int/wer/2012/wer8714.pdf

[2] M. C. Thigpen, C. G. Whitney, N. E. Messonnier, E. R. Zell, R. Lynfield, J. L. Hadler, L. H. Harrison, M. M. Farley, A. Reingold, N. M. Bennett, A. S. Craig, W. Schaffner, A. Thomas, M. M. Lewis, E. Scallan, A. Schuchat and Emerging Infections Programs Network, "Bacterial Meningitis in the United States, 1998-2007," The New England Journal of Medicine, Vol. 361, No. 21, 2011, pp. 2016-2025. doi:10.1056/NEJMoa1005384

[3] M. Deloria-Knoll, K. L. O’Brien, E. Henkle, E. Lee, J. P. Watt, N. McCall and P. Mangtani, "Global Literature Review of Haemophilus influenzae Type b and Streptococcus pneumoniae Invasive Disease among Children Less than Five Years of Age, 1980-2005," 2009.

http://whqlibdoc.who.int/hq/2009/WHO_IVB_09.02_eng. pdf

[4] K. L. O’Brien, L. J. Wolfson, J. P. Watt, E. Henkle, M. Deloria-Knoll, N. McCall, E. Lee, K. Mulholland, O. S. Levine, T. Cherian and Hib and Pneumococcal Global Burden of Disease Study Team, "Burden of Disease Caused by Streptococcus pneumoniae in Children Younger than 5 Years: Global Estimates," Lancet, Vol. 374, No. 9693, 2009, pp. 893-902. doi:10.1016/S0140-6736(09)61204-6

[5] T. Pilishvili, C. Lexau, M. M. Farley, J. Hadler, L. H. 
Harrison, N. M. Bennett, A. Reingold, A. Thomas, W. Schaffner, A. S. Craig, P. J. Smith, B. W. Beall, C. G. Whitney, M. R. Moore and Active Bacterial Core Surveillance/Emerging Infections Program Network, "Sustained Reductions in Invasive Pneumococcal Disease in the Era of Conjugate Vaccine," Journal of Infectious Diseases, Vol. 201, No. 1, 2010, pp. 32-41.

doi:10.1086/648593

[6] D. J. Isaacman, E. D. McIntosh and R. R. Reinert, "Burden of Invasive Pneumococcal Disease and Serotype Distribution among Streptococcus pneumoniae Isolates in Young Children in Europe: Impact of the 7-Valent Pneumococcal Conjugate Vaccine and Considerations for $\mathrm{Fu}-$ ture Conjugate Vaccines," International Journal of Infectious Diseases, Vol. 14, No. 3, 2010, pp. e197-e209.

[7] M. Pulido and F. Sorvillo, "Declining Invasive Pneumococcal Disease Mortality in the United States, 19902005," Vaccine, Vol. 28, No. 4, 2010, pp. 889-892. doi:10.1016/j.vaccine.2009.10.121

[8] E. C. Dinleyici and Z. A. Yargic, "Current Knowledge regarding the Investigational 13-Valent Pneumococcal Conjugate Vaccine," Expert Review of Vaccines, Vol. 8, No. 9, 2009, pp. 977-986. doi:10.1586/erv.09.68

[9] L. A. Hicks, L. H. Harrison, B. Flannery, J. L. Hadler, W. Schaffner, A. S. Craig, D. Jackson, A. Thomas, B. Beall, R. Lynfield, A. Reingold, M. M. Farley and C. G. Whitney, "Incidence of Pneumococcal Disease Due to Non-Pneumococcal Conjugate Vaccine (PCV7) Serotypes in the United States during the Era of Widespread PCV7 Vaccination, 1998-2004," Journal of Infectious Diseases, Vol. 196, No. 9, 2007, pp. 1346-1354. doi:10.1086/521626

[10] E. D. McIntosh and R. R. Reinert, "Global Prevailing and Emerging Pediatric Pneumococcal Serotypes," Expert Review of Vaccines, Vol. 10, No. 1, 2011, pp. 109-129. doi:10.1586/erv.10.145

[11] F. D’Ancona, S. Salmaso, A. Barale, D. Boccia, P. L. Lopalco, C. Rizzo, M. Monaco, M. Massari, V. Demicheli, A. Pantosti and Italian PNC-Euro Working Group, "Incidence of Vaccine Preventable Pneumococcal Invasive Infections and Blood Culture Practices in Italy," Vaccine, Vol. 23, No. 19, 2005, pp. 2494-2500. doi:10.1016/j.vaccine.2004.10.037

[12] L. Tarallo, F. Tancredi, G. Schito, A. Marchese, A. Bella and Italian Pneumonet Group, "Active Surveillance of Streptococcus pneumoniae Bacteremia in Italian Children," Vaccine, Vol. 24, No. 47-48, 2006, pp. 6938-6943. doi:10.1016/j.vaccine.2006.05.012

[13] S. Tardivo, A. Poli, T. Zerman, R. D'Elia, G. Chiamenti, E. Torri, A. Bonetti, E. Pedevilla, P. Pancheri, P. Lubrano, R. Savastano, G. Meneghelli and G. Romano, "Invasive Pneumococcal Infections in Infants up to Three Years of Age: Results of a Longitudinal Surveillance in North-East Italy," Annali di Igiene, Vol. 21, No. 6, 2009, pp. 619628.

[14] P. Durando, P. Crovari, F. Ansaldi, L. Sticchi, C. Sticchi, V. Turello, L. Marensi, R. Giacchino, A. Timitilli, R. Carloni, C. Azzari, G. Icardi and Collaborative Group for Pneumococcal Vaccination in Liguria, "Universal Child- hood Immunisation against Streptococcus pneumoniae: The Five-Year Experience of Liguria Region, Italy," Vaccine, Vol. 27, No. 25-26, 2009, pp. 3459-3462. doi:10.1016/j.vaccine.2009.01.052

[15] A. Marchese, S. Esposito, E. Coppo, G. A. Rossi, A. Tozzi, M. Romano, L. Da Dalt, G. C. Schito and N. Principi, "Detection of Streptococcus Pneumoniae and Identification of Pneumococcal Serotypes by Real-Time Polymerase Chain Reaction Using Blood Samples from Italian children $\leq 5$ Years of Age with Community-Acquired Pneumonia," Microbial Drug Resistance, Vol. 17, No. 3, 2011, pp. 419-424. doi:10.1089/mdr.2011.0031

[16] C. Azzari, M. Moriondo, M. Cortimiglia, C. Valleriani, C. Canessa, G. Indolfi, S. Ricci, F. Nieddu, M. de Martino, M. Resti and the Italian Group for the Study of Invasive Pneumococcal Disease, "Potential Serotype Coverage of Three Pneumococcal Conjugate Vaccines against Invasive Pneumococcal Infection in Italian Children," Vaccine, Vol. 30, No. 16, 2012, pp. 2701-2705. doi:10.1016/j.vaccine.2011.12.008

[17] Italian Ministry of Health, "National Vaccine Plan 20122014, [Piano Nazionale Prevenzione Vaccinale 20122014, in Italian]," 2012.

http://www.salute.gov.it/imgs/C_17_pubblicazioni_1721_ allegato.pdf

[18] L. J. Baraff, J. W. Bass, G. R. Fleisher, J. O. Klein, G. H. McCracken Jr., K. R. Powell and D. L. Schriger, "Practice Guideline for the Management of Infants and Children 0 to 36 Months of Age with Fever without Source; Agency for Health Care Policy and Research," Annals of Emergency Medicine, Vol. 22, No. 7, 1993, pp. 1198-2210. doi:10.1016/S0196-0644(05)80991-6

[19] Centers for Disease Control and Prevention, "Case Definitions for Infectious Conditions under Public Health Surveillance," MMWR Recommendations and Reports, Vol. 46, No. 10, 1997, pp. 1-55.

[20] Centers for Disease Control and Prevention, "Manual for the Surveillance of Vaccine-Preventable Diseases. Chapter 11: Pneumococcal," 4th Edition, Centers for Disease Control and Prevention, Atlanta, 2008.

http://www.cdc.gov/vaccines/pubs/surv-manual/chpt11-p neumo.html

[21] World Health Organization, "International Classification of Diseases," 9th Revision, World Health Organization, Geneva, 1980.

[22] Clinical and Laboratory Standards Institute, "Performance Standards for Antimicrobial Susceptibility Testing; Nineteenth Informational Supplement; M100-S19," Clinical and Laboratory Standards Institute, Wayne, 2009.

[23] S. I. Aguiar, M. J. Brito, J. Gonçalo-Marques, J. MeloCristino and M. Ramirez, "Serotypes 1, 7F and 19A Became the Leading Causes of Pediatric Invasive Pneumococcal Infections in Portugal after 7 Years of Heptavalent Conjugate Vaccine Use," Vaccine, Vol. 28, No. 32, 2010, pp. 5167-5173. doi:10.1016/j.vaccine. 2010.06 .008

[24] B. A. Kendall, K. K. Dascomb, R. R. Mehta, E. O. Mason, K. Ampofo, D. J. Pombo, A. T. Pavia and C. L. Byington, "Streptococcus pneumoniae Serotypes in Utah Adults at 
the End of the PCV7 Era," Vaccine, Vol. 29, No. 4, 2011, pp. 9123-9126. doi:10.1016/j.vaccine.2011.10.009

[25] Istituto Superiore di Sanità, ICONA Working Group, "ICONA 2008: National Vaccination Coverage Survey among Children and Adolescents," 2009.

http://www.iss.it/binary/publ/cont/09_29_web.pdf

[26] Centers for Disease Control and Prevention, "Active Bacterial Core Surveillance Report, Emerging Infections Program Network, Streptococcus pneumoniae, 2010," 2012. http://www.cdc.gov/abcs/reports-findings/survreports/spn eu10.html

[27] Istituto Superiore di Sanità, "Data on Surveillance Data of Invasive Bacterial Diseases-Updating February 24, 2012, [Dati di Sorveglianza Delle Malattie Batteriche InvasiveAggiornati al 24 Febbraio 2012, in Italian],” 2010. http://www.simi.iss.it/dati.htm

[28] K. K. Hsu, K. M. Shea, A. E. Stevenson, S. I. Pelton and Massachusetts Department of Public Health, "Changing Serotypes Causing Childhood Invasive Pneumococcal Disease: Massachusetts, 2001-2007," The Pediatric Infectious Disease Journal, Vol. 29, No. 4, 2010, pp. 289-293.

[29] Y. C. Hsieh, P. Y. Lin, C. H. Chiu, Y. C. Huang, K. Y. Chang, C. H. Liao, N. C. Chiu, Y. C. Chuang, P. Y .Chen,

\section{Appendix A. List of Participant Hospitals and Reference Laboratory}

Department of Pediatrics, San Paolo Hospital, University of Milan, Milan: Marcello Giovannini, Enrica Riva, Filippo Salvini. Department of Pediatrics and Neonatology, "Sant'Antonio Abate" Hospital, Cantù, Italy: Mario Barbarini. Department of Pediatrics, Fatebenefratelli Hospital, Milan, Italy: Luca Bernardo. Department of Pediatrics, Ospedale di Desio, Desio, Italy: Roberto Besana. Department of Pediatrics, Ospedale di Vizzolo Pedabissi, A. O. di Melegnano, Vizzolo Predabissi, Italy: Gianluigi
S. C. Chang, J. W. Liu, M. Y. Yen, J. H. Wang, C. Y. Liu and T. Y. Lin, "National Survey of Invasive Pneumococcal Diseases in Taiwan under Partial PCV7 Vaccination in 2007: Emergence of Serotype 19A with High Invasive Potential," Vaccine, Vol. 27, No. 40, 2009, pp. 55135518. doi:10.1016/j.vaccine.2009.06.091

[30] S. L. Kaplan, W. J. Barson, P. L. Lin, S. H. Stovall, J. S. Bradley, T. Q. Tan, J. A. Hoffman, L. B. Givner and E. O. Mason Jr., "Serotype 19A Is the Most Common Serotype Causing Invasive Pneumococcal Infections in Children," Pediatrics, Vol. 125, No. 3, 2010, pp. 429-436. doi:10.1542/peds.2008-1702

[31] C. G. Grijalva and S. I. Pelton, “A Second-Generation Pneumococcal Conjugate Vaccine for Prevention of Pneumococcal Diseases in Children," Current Opinion in Pediatrics, Vol. 23, No. 1, 2011, pp. 98-104. doi:10.1097/MOP.0b013e328341d1f5

[32] T. Avni, N. Mansur, L. Leibovici and M. Paul, "PCR Using Blood for Diagnosis of Invasive Pneumococcal Disease: Systematic Review and Meta-Analysis," Journal of Clinical Microbiology, Vol. 48, No. 2, 2010, pp. 489496. doi:10.1128/JCM.01636-09

Gargantini. Department of Pediatrics, Sant'Anna Hospital, Como, Italy: Roberto Longhi. Department of Pediatrics, Ospedale di Cernusco sul Naviglio, A. O. di Melegnano, Cernusco sul Naviglio, Italy: Amilcare Rottoli. Department of Pediatrics, Ospedale di Carate Brianza, Carate Brianza, Italy: Andrea Sterpa, Department of Pediatrics, "Luigi Sacco" Hospital, University of Milan, Milan, Italy: Gianvincenzo Zuccotti. Pneumococcal Reference Laboratory in Lombardy, Milan, Italy: Laura Daprai and Maria Laura Garlaschi. 